\title{
Local well-posedness for 3D micropolar fluid system in Besov-Morrey spaces
}

\author{
Lucas C. F. Ferreira \\ IMECC - UNICAMP \\ 13083-859, Campinas, SP \\ E-mail: lcff@ime.unicamp.br.
}

Juliana C. Precioso,

Depto Matemática, IBILCE, UNESP,

15054-000, São José do Rio Preto, SP

E-mail: precioso@ibilce.unesp.br

Resumo: We show a local-in-time existence result for the 3D micropolar fluid system in the framework of Besov-Morrey spaces. The initial data class is larger than the previous ones and contains strongly singular functions and measures.

Palavras-chave: Micropolar fluids, well-posedness, Besov-Morrey spaces

\section{Introduction}

In this work we are concerned with a system of equations describing a viscous incompressible homogeneous micropolar fluid filling the whole space $\mathbb{R}^{3}$ and with density equal to one. This system was introduced by A.C. Eringer in [1] and can be used to model the behavior of some fluids under micro-rotation effects caused by rigid suspended particles in a viscous medium. Examples of those are polymeric fluids, animal blood, liquid crystals, ferro-liquids, and many others. These fluids cannot be modeled by only using Navier-Stokes equations due to the important role played by their microstructures which make them to be non-newtonian fluids with nonsymmetric stress tensor.

The initial value problem (IVP) for the micropolar system reads as

$$
\begin{gathered}
\frac{\partial u}{\partial t}-(\chi+\nu) \Delta u+u \cdot \nabla u+\nabla \pi-2 \chi \nabla \times \omega=0, \quad x \in \mathbb{R}^{3}, t>0, \\
\frac{\partial \omega}{\partial t}-\mu \Delta \omega+u \cdot \nabla \omega+4 \chi \omega-\kappa \nabla(\nabla \cdot \omega)-2 \chi \nabla \times u=0, \quad x \in \mathbb{R}^{3}, t>0, \\
\nabla \cdot u=0, \quad x \in \mathbb{R}^{3}, t>0, \\
\left.u\right|_{t=0}=u_{0}, \nabla \cdot u_{0}=0 \text { and }\left.\omega\right|_{t=0}=\omega_{0}, \quad x \in \mathbb{R}^{3},
\end{gathered}
$$

where the vector $u(x, t)$ is the linear velocity of the fluid, the scalar $\pi(x, t)$ represents the pressure, and $\omega(x, t)$ is the rotation velocity field of particles. The symbols $\nabla \cdot u$ and $\nabla \times u$ stand respectively for the divergence and rotational of the field $u$. The equations (1.1)-(1.3) are completed with Dirichlet conditions at infinity, that is, $u, \omega \rightarrow 0$ as $|x| \rightarrow \infty$. The fluid physical characteristics are determined by the constants $\nu, \chi, \kappa, \mu$, where $\nu$ denotes the Newtonian viscosity and the parameters $\chi, \kappa, \mu$ are viscosities related to the rotation field of particles $\omega$ (see [4]). The data $u_{0}$ and $\omega_{0}$ are respectively the initial linear and rotation velocity. For simplicity of exposition, we assume $\chi=\nu=1 / 2$ and $\kappa=\mu=1$. 
The micro-rotation influence on velocity field $u$ disappears when $\chi=0$ or $\omega=0$ in (1.1)-(1.4), and then the 3D Navier-Stokes equations (3DNS) and their newtonian structure is recovered. We have a rich literature about existence of solutions for 3DNS in several frameworks, such as Lebesgue space $L^{p}$, weak $-L^{p}(p \geq 3)$, Morrey spaces $M_{p, \lambda}(\lambda \in[0,3), p \geq 3-\lambda), P M^{a}$ $(2 \leq a<3)$, Besov spaces $B_{p, \infty}^{-k}\left(0<k \leq 1-\frac{3}{p}\right), B M O_{R}^{-1}(0<R \leq \infty)$, Besov-Morrey spaces $N_{p, \lambda, \infty}^{-s}$ with $p \in[1, \infty), 0 \leq \lambda<3$ and $0<s \leq 1-\frac{3-\lambda}{p}$, and some others. For $p=1$, there is no an inclusion relation between $B M O_{R}^{-1}$ and $N_{p, \lambda, \infty}^{-s}$ (see e.g. [5, p.18]) and they are maximal classes for local-in-time existence in the whole space $\mathbb{R}^{3}$.

It is natural to wonder which of those existence results for 3DNS could be extended for a fluid under the effect of micro-rotations and with a non-newtonian structure.

The goal of this work is to prove a local well-posedness result in a new setting whose initial data class is maximal for existence of solutions for (1.1)-(1.4). We consider the framework of Besov-Morrey spaces $N_{p, \lambda, \infty}^{-s}$ which contain strongly singular functions and measures supported in either points (Diracs), filaments or surfaces (see e.g. [2, Remark 3.3] for more details). This class is larger than the previous ones in view of the continuous inclusions

$$
L^{p} \subset \text { weak- } L^{p} \subset B_{q, \infty}^{-k} \subset N_{r, \lambda, \infty}^{-s} \text { and } P M^{a} \subset B_{q, \infty}^{-k} \subset N_{r, \lambda, \infty}^{-s},
$$

when $\frac{3}{p}=3-a=k+\frac{n}{q}=s+\frac{n-\lambda}{r}$ ( in other words, the spaces in (1.5) have the same scaling).

\section{Preliminaries}

\section{$2.1 \quad$ Function spaces and definitions}

For $1 \leq p<\infty$ and $0 \leq \lambda<n$, the local Morrey space $M_{p, \lambda}=M_{p, \lambda}\left(\mathbb{R}^{n}\right)$ is defined as

$$
M_{p, \lambda}=\left\{f \in L_{l o c}^{p}\left(\mathbb{R}^{n}\right):\|f\|_{p, \lambda}<\infty\right\}
$$

where

$$
\|f\|_{p, \lambda}=\sup _{x_{0} \in \mathbb{R}^{n}, 0<R \leq 1}\left(R^{-\frac{\lambda}{p}}\|f\|_{L^{p}\left(B_{R}\left(x_{0}\right)\right)}\right)
$$

and $B_{R}\left(x_{0}\right) \subset \mathbb{R}^{n}$ is the open ball with center $x$ and radius $R$. The space $M_{p, \lambda}$ endowed with $\|\cdot\|_{p, \lambda}$ is a Banach space. When $p=1, M_{p, \lambda}$ should be understood as a space of Radon measures and the $L^{p}$-norm in (2.2) as the total variation of the measure $f$ computed on $B_{R}\left(x_{0}\right)$.

Hölder inequality holds true in the framework of Morrey spaces. Precisely, if $1 \leq p_{i} \leq \infty$ and $0 \leq \lambda_{i}<n$ with $\frac{1}{p_{3}}=\frac{1}{p_{1}}+\frac{1}{p_{2}}$ and $\frac{\lambda_{3}}{p_{3}}=\frac{\lambda_{1}}{p_{1}}+\frac{\lambda_{2}}{p_{2}}$, then

$$
\|f g\|_{p_{3}, \lambda_{3}} \leq\|f\|_{p_{1}, \lambda_{1}}\|g\|_{p_{2}, \lambda_{2}} .
$$

Recalling the notation $M_{p, \lambda}^{s}=(-\Delta)^{-s / 2} M_{p, \lambda}$ for Sobolev Morrey spaces, the inhomogeneous Besov-Morrey space $N_{p, \lambda, q}^{s}$ is the following interpolation space

$$
\left(M_{p, \lambda}^{s_{1}}, M_{p, \lambda}^{s_{2}}\right)_{\theta, q}=N_{p, \lambda, q}^{s},
$$

where $\theta \in(0,1)$ and $s=(1-\theta) s_{1}+\theta s_{2}$ with $s_{1} \neq s_{2}$. In view of $(2.4)$, the reiteration theorem implies that

$$
\left(N_{p, \lambda, q_{1}}^{s_{1}}, N_{p, \lambda, q_{2}}^{s_{2}}\right)_{\theta, q}=N_{p, \lambda, q}^{s},
$$

where $1 \leq q, q_{1}, q_{2} \leq \infty$ with $q^{-1}=(1-\theta) q_{1}^{-1}+\theta q_{2}^{-1}$ and $\theta \in(0,1)$.

The space $N_{p, \lambda, q}^{s}$ can be characterized via norms based on dyadic decompositions. For that matter, let $D_{0} \stackrel{\rho}{=}\left\{\xi \in \mathbb{R}^{n}:|\xi|<1\right\}$ and let $\varphi_{0} \in \mathcal{S}\left(\mathbb{R}^{n}\right)$ be such that $\operatorname{supp}\left(\widehat{\varphi}_{0}\right) \subset D_{0}$ and 
$\widehat{\varphi}_{0}(\xi)=1$ if $|\xi| \leq \frac{2}{3}$. Define $\varphi_{k}=2^{k n} \varphi_{0}\left(2^{k} \xi\right)$ and $\psi_{k}=\varphi_{k+1}-\varphi_{k}$ for all $k \in\{0\} \cup \mathbb{N}$. Then $\widehat{\varphi}_{k}(\xi)=\widehat{\varphi}_{0}\left(2^{-k} \xi\right), \widehat{\psi}_{k}=\widehat{\varphi}_{k+1}-\widehat{\varphi}_{k}, \operatorname{supp}\left(\widehat{\psi}_{k}\right) \subset\left\{\xi \in \mathbb{R}^{n}: 2^{k-1}<|\xi|<2^{k+1}\right\}$ and

$$
\widehat{\varphi}_{0}+\sum_{k=0}^{\infty} \widehat{\psi}_{k}(\xi)=1, \text { for all } \xi
$$

For $f \in \mathcal{S}^{\prime}$, consider the quantity $\|f\|_{N_{p, \lambda, q}^{s}}$ given by

$$
\left\{\begin{array}{l}
\left\|\varphi_{0} * f\right\|_{p, \lambda}+\left(\sum_{k=0}^{\infty}\left(2^{k s}\left\|\psi_{k} * f\right\|_{p, \lambda}\right)^{q}\right)^{\frac{1}{q}}, \text { if } 1 \leq p \leq \infty, 1 \leq q<\infty, s \in \mathbb{R} . \\
\left\|\varphi_{0} * f\right\|_{p, \lambda}+\sup _{k \in\{0\} \cup \mathbb{N}}\left(2^{k s}\left\|\psi_{k} * f\right\|_{p, \lambda}\right), \text { if } 1 \leq p \leq \infty, q=\infty, s \in \mathbb{R} .
\end{array}\right.
$$

We have that

$$
N_{p, \lambda, q}^{s}=\left\{f \in \mathcal{S}^{\prime}\left(\mathbb{R}^{n}\right):\|f\|_{N_{p, \lambda, q}^{s}}<\infty\right\}
$$

and the pair $\left(N_{p, \lambda, q}^{s},\|\cdot\|_{N_{p, \lambda, q}^{s}}\right)$ is a Banach space. The inclusion $N_{p, \lambda, q_{1}}^{s} \subset N_{p, \lambda, q_{2}}^{s}$ is continuous for $1 \leq q_{1} \leq q_{2} \leq \infty$, and

$$
N_{p, \lambda, 1}^{0} \subset M_{p, \lambda} \subset N_{p, \lambda, \infty}^{0},
$$

for all $1 \leq p<\infty$ and $0 \leq \lambda<n$. Finally we recall the Sobolev type embedding

$$
N_{p_{2}, \lambda, q_{2}}^{s_{2}} \subset N_{p_{1}, \lambda, q_{1}}^{s_{1}}, \quad \text { for } p_{1}>p_{2} \text { and } s_{1}-\frac{n-\lambda}{p_{1}}=s_{2}-\frac{n-\lambda}{p_{2}} .
$$

The following lemma can be found in [6] and gives estimates for some multiplier operators acting in $N_{p, \lambda, r}^{s}$.

Lemma 2.1. Let $m, s \in \mathbb{R}, 1 \leq p<\infty, 0 \leq \lambda<n$ and $1 \leq r \leq \infty$. Let $P(\xi) \in C^{[n / 2]+1}\left(\mathbb{R}^{n}\right)$ where [.] stands for the greatest integer function. Assume that there is $A>0$ such that

$$
\left|\frac{\partial^{|\alpha|} P}{\partial \xi^{\alpha}}(\xi)\right| \leq A\langle\xi\rangle^{m-|\alpha|} \text {, where }\langle\xi\rangle=\left(1+|\xi|^{2}\right)^{1 / 2},
$$

for all $\xi \in \mathbb{R}^{n}$ and $|\alpha| \leq[n / 2]+1$. Then the operator $P(D)$ is bounded from $N_{p, \lambda, r}^{s}$ to $N_{p, \lambda, r}^{s-m}$ and satisfies the estimate

$$
\|P(D) u\|_{N_{p, \lambda, r}^{s-m}} \leq C A\|u\|_{N_{p, \lambda, r}^{s}},
$$

where $C>0$ is a constant depending only on $s, m, p, \lambda$.

\subsection{Mild solutions}

Recall that we are considering $\chi=\nu=1 / 2$ and $\kappa=\mu=1$ in (1.1)-(1.4). After applying the Leray projector in (1.1), we obtain the system

$$
\left\{\begin{array}{l}
\partial_{t} u-\Delta u-\nabla \times \omega+\mathbb{P}(u \cdot \nabla u)=0 \\
\partial_{t} \omega-\Delta \omega+u \cdot \nabla \omega+2 \omega-\nabla(\nabla \cdot \omega)-\nabla \times u=0 \\
\left.u\right|_{t=0}=u_{0}, \nabla \cdot u_{0}=0, \text { and }\left.\omega\right|_{t=0}=\omega_{0}
\end{array} .\right.
$$

The linearized one associated to (2.10) is

$$
\left\{\begin{array}{l}
\partial_{t} u-\Delta u-\nabla \times \omega=0 \\
\partial_{t} \omega-\Delta \omega+2 \omega-\nabla(\nabla \cdot \omega)-\nabla \times u=0 \\
\left.u\right|_{t=0}=u_{0}, \nabla \cdot u_{0}=0, \text { and }\left.\omega\right|_{t=0}=\omega_{0}
\end{array} .\right.
$$


Proceeding as in [3], we apply the Fourier transform in (2.11) and use the notation $y=[u, \omega]$ in order to obtain

$$
\left\{\begin{array}{l}
\partial_{t} \hat{y}+A(\xi) \hat{y}=0 \\
\hat{y}(\xi, 0)=\left(\hat{u}_{0}, \hat{\omega}_{0}\right)
\end{array},\right.
$$

where

$$
A(\xi)=\left(\begin{array}{cc}
|\xi|^{2} I & B(\xi) \\
B(\xi) & R(\xi)+\left(|\xi|^{2}+2\right) I
\end{array}\right)=A_{1}(\xi)+A_{2}(\xi)+A_{3}(\xi)
$$

with

$$
A_{1}=\left(\begin{array}{cc}
|\xi|^{2} I & 0 \\
0 & \left(|\xi|^{2}+2\right) I
\end{array}\right), A_{2}=\left(\begin{array}{cc}
0 & B(\xi) \\
B(\xi) & 0
\end{array}\right), A_{3}=\left(\begin{array}{cc}
0 & 0 \\
0 & R(\xi)
\end{array}\right)
$$

and

$$
R(\xi)=\left(\begin{array}{ccc}
\xi_{1}^{2} & \xi_{1} \xi_{2} & \xi_{1} \xi_{3} \\
\xi_{1} \xi_{2} & \xi_{2}^{2} & \xi_{2} \xi_{3} \\
\xi_{1} \xi_{3} & \xi_{2} \xi_{3} & \xi_{3}^{2}
\end{array}\right) \quad \text { and } \quad B(\xi)=i\left(\begin{array}{ccc}
0 & \xi_{3} & -\xi_{2} \\
-\xi_{3} & 0 & -\xi_{1} \\
\xi_{2} & -\xi_{1} & 0
\end{array}\right)
$$

For each $t \geq 0$, we define the operator $G_{A}(t)$ via Fourier variables by

$$
\widehat{G_{A}(t) y_{0}}(\xi)=e^{-A(\xi) t} \hat{y}_{0}
$$

where $A(\xi)$ has been defined in (2.13). From [3, inequality (20), p.1430] with $\gamma=\beta=1$, we have the pointwise estimate

$$
\left|e^{-t A(\xi)}\right| \leq C e^{-|\xi|^{2} t} .
$$

Notice that the family $\left\{G_{A}(t)\right\}_{t \geq 0}$ is formally a semigroup and $y=[u, \omega]=G_{A}(t) y_{0}$ is the solution of the linearized problem (2.11). Then, according to Duhamel's principle, the problem (2.10) is formally equivalent to the integral system

$$
y(x, t)=G_{A}(t) y_{0}-\int_{0}^{t} G_{A}(t-s) \mathbb{P} \nabla \cdot(u \otimes y) d s,
$$

where

$$
\mathbb{P} \nabla \cdot(u \otimes y)=[\mathbb{P} \nabla \cdot(u \otimes u), \nabla \cdot(u \otimes \omega)] .
$$

Throughout this paper, solutions of (2.17) are called mild ones for (1.1)-(1.4) (or for (2.10)).

\section{Results}

The purpose of this section is to state our existence result for the Cauchy problem (1.1)-(1.4). Given a vector space $X \subset\left(\mathcal{S}^{\prime}\left(\mathbb{R}^{3}\right)\right)^{3}$, we denote $X^{\sigma}$ as the set of all $u \in X$ such that $\operatorname{div}(u)=0$ in $\mathcal{S}^{\prime}\left(\mathbb{R}^{3}\right)$.

Let $\eta, \beta>0,1 \leq q<\infty, 0 \leq \lambda<3$ and $\eta_{0}=\frac{1}{2}-\frac{3-\lambda}{4 q}$. Many times, spaces of scalar and vector functions will be denoted abusively in the same way, e.g. $N_{q, \lambda, \infty}^{-\beta}=\left(N_{q, \lambda, \infty}^{-\beta}\right)^{3}$. Also, $N_{q, \lambda, \infty}^{-\beta, \sigma}$ stands for $\left(\left(N_{q, \lambda, \infty}^{-\beta}\right)^{3}\right)^{\sigma}$.

We will look for local-in-time mild solutions $[u(x, t), \omega(x, t)]$ in the class $\mathcal{X}_{T}$ defined by

$$
\left\{[u, \omega] \in B C\left((0, T) ; N_{q, \lambda, \infty}^{-\beta, \sigma} \times N_{q, \lambda, \infty}^{-\beta}\right):\left[t^{\eta} u, t^{\eta} \omega\right] \in B C\left((0, T) ;\left(M_{2 q, \lambda}\right)^{2}\right)\right\},
$$

which is a Banach space with norm

$$
\|[u, \omega]\|_{\mathcal{X}_{T}}=\sup _{0<t<T}\|[u(\cdot, t), \omega(\cdot, t)]\|_{N_{q, \lambda, \infty}^{-\beta}}+\sup _{0<t<T} t^{\eta}\|[u(\cdot, t), \omega(\cdot, t)]\|_{2 q, \lambda},
$$


where $\|[\cdot, \cdot]\|_{N_{q, \lambda, \infty}^{-\beta}}=\|[\cdot, \cdot]\|_{\left(N_{q, \lambda, \infty}^{-\beta}\right)^{2}}$ and $\|[\cdot, \cdot]\|_{2 q, \lambda}=\|[\cdot, \cdot]\|_{\left(M_{2 q, \lambda}\right)^{2}} \cdot$

The initial data is taken in the class

$$
\left[u_{0}, \omega_{0}\right] \in N_{q, \lambda, \infty}^{-\beta, \sigma} \times N_{q, \lambda, \infty}^{-\beta} .
$$

We are ready to state our well-posedness result.

Theorem 3.1. Assume that $1 \leq q<\infty, 0 \leq \lambda<3,0<\beta<1-\frac{3-\lambda}{q}$ and $\frac{\beta}{2}+\frac{3-\lambda}{4 q} \leq \eta<$ $\min \left\{\eta_{0}, \frac{\beta-1}{4}+\frac{1}{2}\right\}$. Suppose that the initial data $\left[u_{0}, \omega_{0}\right]$ belongs to the class (3.3).

(i) (Existence and uniqueness) There exists $T>0$ such that (1.1)-(1.4) has a mild solution $y=[u, \omega] \in \mathcal{X}_{T}$, which is the unique one in a suitable closed ball $\mathcal{B}_{r}$ of $\mathcal{X}_{T}$ whose radius $r>0$ increases with the size of the initial data.

(ii) (Continuous dependence) The solution $[u, \omega]$ depends continuously on initial data $\left[u_{0}, \omega_{0}\right]$.

\section{Proofs}

In order to perform a contraction argument, we need to obtain estimates in Besov-Morrey spaces for the linear and bilinear terms of the integral equation (2.17). Let us start with core estimates for the semigroup $\left\{G_{A}(t)\right\}_{t \geq 0}$.

\subsection{Estimates for $G_{A}(t)$ in $N_{p, \lambda, r}^{s}$-spaces}

The next lemma gives estimates for $\left\{G_{A}(t)\right\}_{t \geq 0}$ on spaces $N_{p, \lambda, r}^{s}$. In particular, these operators are well defined in the setting of $N_{p, \lambda, r}^{s}$-spaces.

Lemma 4.1. Let $s, \beta \in \mathbb{R}, s \leq \beta, 1 \leq q \leq \infty, 1 \leq r \leq \infty$ and $0 \leq \lambda<3$. There exists a constant $C>0$ such that

$$
\left\|G_{A}(t) y\right\|_{N_{q, \lambda, r}^{\beta}} \leq C(1+t)^{2}\left(1+t^{\frac{s-\beta}{2}}\right) \quad\|y\|_{N_{q, \lambda, r}^{s}},
$$

for all $t>0$ and $y \in N_{q, \lambda, r}^{s}$. Furthermore, if $s<\beta$, then

$$
\left\|G_{A}(t) y\right\|_{N_{q, \lambda, 1}^{\beta}} \leq C(1+t)^{2}\left(1+t^{\frac{s-\beta}{2}}\right) \quad\|y\|_{N_{q, \lambda, \infty}^{s}},
$$

for all $t>0$ and $y \in N_{q, \lambda, \infty}^{s}$.

\subsection{Bilinear estimates}

In the remainder of this work, we use the following notation for the bilinear operator appearing in $(2.17)$

$$
B\left(y_{1}, y_{2}\right)=-\int_{0}^{t} G_{A}(t-s) \mathbb{P} \nabla \cdot\left(u_{1} \otimes y_{2}\right) d s,
$$

where $y_{1}=\left[u_{1}, \omega_{1}\right], y_{2}=\left[u_{2}, \omega_{2}\right]$.

Lemma 4.2. Let $0<T<\infty$. Under the hypotheses of Theorem 3.1, there exist constants $K_{1}=K_{1}(T), K_{2}=K_{2}(T)>0$ such that

$$
\begin{aligned}
& \sup _{0<t<T}\left\|B\left(y_{1}, y_{2}\right)\right\|_{N_{q, \lambda, \infty}^{-\beta}} \leq K_{1} \sup _{0<t<T} t^{\eta}\left\|y_{1}(\cdot, t)\right\|_{2 q, \lambda} \sup _{0<t<T} t^{\eta}\left\|y_{2}(\cdot, t)\right\|_{2 q, \lambda}, \\
& \sup _{0<t<T} t^{\eta}\left\|B\left(y_{1}, y_{2}\right)\right\|_{2 q, \lambda} \leq K_{2} \sup _{0<t<T} t^{\eta}\left\|y_{1}(\cdot, t)\right\|_{2 q, \lambda} \sup _{0<t<T} t^{\eta}\left\|y_{2}(\cdot, t)\right\|_{2 q, \lambda},
\end{aligned}
$$

for all $y_{1}, y_{2} \in \mathcal{X}_{T}$. Moreover, $K_{i}(T) \rightarrow 0^{+}$as $T \rightarrow 0^{+}$, for $i=1,2$. 


\subsection{Proof of Theorem 3.1.}

Part (i): Recall the notation $B(\cdot, \cdot)$ in (4.3). Lemma 4.2 yields

$$
\begin{aligned}
\|B(y, z)\|_{\mathcal{X}_{T}} & =\sup _{0<t<T}\|B(y, z)\|_{N_{q, \lambda, \infty}^{-\beta}}+\sup _{0<t<T} t^{\eta}\|B(y, z)\|_{2 q, \lambda} \\
& \leq\left(K_{1}(T)+K_{2}(T)\right)\left(\sup _{0<t<T} t^{\eta}\|y(\cdot, t)\|_{2 q, \lambda} \sup _{0<t<T} t^{\eta}\|z(\cdot, t)\|_{2 q, \lambda}\right) \\
& \leq K(T)\|y\|_{\mathcal{X}_{T}}\|z\|_{\mathcal{X}_{T}},
\end{aligned}
$$

where $K(T)=K_{1}(T)+K_{2}(T)$.

Let $\tau_{q, \lambda}=\frac{3-\lambda}{q}$. Using the inclusion $N_{q, \lambda, 1}^{0} \hookrightarrow M_{q, \lambda}$ (see (2.8)), Sobolev type embedding (2.9), and Lemma 4.1, we obtain

$$
\begin{aligned}
\left\|G_{A}(t) y_{0}\right\|_{\mathcal{X}_{T}}= & \sup _{0<t<T}\left\|G_{A}(t) y_{0}\right\|_{N_{q, \lambda, \infty}^{-\beta}}+\sup _{0<t<T} t^{\eta}\left\|G_{A}(t) y_{0}\right\|_{2 q, \lambda} \\
\leq & 2 C(1+T)^{2} \sup _{0<t<T}\left\|y_{0}\right\|_{N_{q, \lambda, \infty}^{-\beta}}+C \sup _{0<t<T} t^{\eta}\left\|G_{A}(t) y_{0}\right\|_{N_{2 q, \lambda, 1}^{0}} \\
\leq & 2 C(1+T)^{2} \sup _{0<t<T}\left\|y_{0}\right\|_{N_{q, \lambda, \infty}^{-\beta}}+C \sup _{0<t<T} t^{\eta}\left\|G_{A}(t) y_{0}\right\|_{N_{q, \lambda, 1}} \\
\leq & 2 C(1+T)^{2} \sup _{0<t<T}\left\|y_{0}\right\|_{N_{q, \lambda, \infty}^{-\beta}} \\
& +C \sup _{0<t<T} t^{\eta}(1+t)^{2}\left(1+t^{-\frac{\tau_{q, \lambda}}{4}-\frac{\beta}{2}}\right)\left\|y_{0}\right\|_{N_{q, \lambda, \infty}^{-\beta}} \\
\leq & C(1+T)^{2}\left(2+\left(T^{\eta}+T^{\eta-\frac{\tau_{q, \lambda}}{4}-\frac{\beta}{2}}\right)\right)\left\|y_{0}\right\|_{N_{q, \lambda, \infty}^{-\beta}} \\
= & C_{T}\left\|y_{0}\right\|_{N_{q, \lambda, \infty}^{-\beta}},
\end{aligned}
$$

because $\eta \geq \frac{\beta}{2}+\frac{\tau_{q, \lambda}}{4}$. Consider the map $\Phi$ defined by

$$
\Phi(y)=G_{A}(t) y_{0}+B(y, y) .
$$

Let $T>0$ and $\mathcal{B}_{r}=\left\{y \in \mathcal{X}_{T} ;\|u\|_{\mathcal{X}_{T}} \leq 2 r\right\}$ where

$$
r=C_{T}\left\|y_{0}\right\|_{N_{q, \lambda, \infty}^{-\beta}},
$$

and $C_{T}$ is as in (4.7). Since $K_{i}(T) \rightarrow 0^{+}$as $T \rightarrow 0^{+}$and $K(T)=K_{1}(T)+K_{2}(T)$, we can choose $T>0$ such that

$$
\begin{aligned}
4 K(T) r & =4 K(T) C_{T}\left\|y_{0}\right\|_{N_{q, \lambda, \infty}^{-\beta}} \\
& =C\left\|y_{0}\right\|_{N_{q, \lambda, \infty}^{-\beta}} 4(1+T)^{2}\left(2+\left(T^{\eta}+T^{\eta-\frac{\tau_{q, \lambda}}{4}-\frac{\beta}{2}}\right)\right) K(T)<1 .
\end{aligned}
$$

It follows from bilinearity and (4.6) that

$$
\begin{aligned}
\|\Phi(y)-\Phi(z)\|_{\mathcal{X}_{T}} & =\|B(y, y)-B(z, z)\|_{\mathcal{X}_{T}} \\
& \leq K\|y-z\|_{\mathcal{X}_{T}}\left(\|y\|_{\mathcal{X}_{T}}+\|z\|_{\mathcal{X}_{T}}\right) \\
& \leq 4 r K(T)\|y-z\|_{\mathcal{X}_{T}},
\end{aligned}
$$

for all $y, z \in \mathcal{B}_{r}$. Also, using the inequality (4.10) with $z=0$, we get

$$
\begin{aligned}
\|\Phi(y)\|_{\mathcal{X}_{T}} & \leq\|\Phi(0)\| \mathcal{X}_{T}+\|\Phi(y)-\Phi(0)\|_{\mathcal{X}_{T}} \\
& \leq\left\|G_{A}(t) u_{0}\right\|_{\mathcal{X}_{T}}+K\|y\|_{\mathcal{X}_{T}}^{2} \\
& \leq r+4 r^{2} K(T) \leq 2 r, \text { for } y \in \mathcal{B}_{r},
\end{aligned}
$$


because $4 K(T) r<1$. The estimates (4.11) and (4.12) show that $\Phi: \mathcal{B}_{r} \rightarrow \mathcal{B}_{r}$ is a contraction and has a unique fixed point in $\mathcal{B}_{r}$. This one is the unique solution $y(x, t)$ for the integral equation (2.17) satisfying $\|y\|_{\mathcal{X}_{T}} \leq 2 r$.

Part (ii): Let $y_{1}, y_{2}$ be two solutions obtained in item (i) with respective data $y_{0,1}, y_{0,2}$ and existence times $T_{1}, T_{2}$. We have that $\left\|y_{1}\right\|_{\mathcal{X}_{T_{1}}} \leq 2 r_{1}$ and $\left\|y_{2}\right\|_{\mathcal{X}_{T_{2}}} \leq 2 r_{2}$, where $r_{i}=C_{T_{i}}\left\|y_{i}\right\|_{N_{q, \lambda, \infty}^{-\beta}}$ (see (4.9)) and $0<4 K\left(T_{i}\right) r_{i}<1$. Since $K(T)$ decreases with $T>0$, we have that $0<4 K(T) r_{i}<$ 1 , for $i=1,2$, and $T=\min \left\{T_{1}, T_{2}\right\}$. Thus, taking $r=\max \left\{r_{1}, r_{2}\right\}$, note that $0<4 K(T) r<1$.

Now, using (4.11), it follows that

$$
\begin{aligned}
\left\|y_{1}-y_{2}\right\|_{\mathcal{X}_{T}} & =\left\|G_{A}(t) y_{0,1}-G_{A}(t) y_{0,2}+B\left(y_{1}, y_{1}\right)-B\left(y_{2}, y_{2}\right)\right\|_{\mathcal{X}_{T}} \\
& \leq\left\|G_{A}(t)\left(y_{0,1}-y_{0,2}\right)\right\|_{\mathcal{X}_{T}}+K(T)\left\|y_{1}-y_{2}\right\| \mathcal{X}_{T}\left(\left\|y_{1}\right\|_{\mathcal{X}_{T}}+\left\|y_{2}\right\|_{\mathcal{X}_{T}}\right) \\
& \leq C_{T}\left\|y_{0,1}-y_{0,2}\right\|_{N_{q, \lambda, \infty}^{-\beta}}+2\left(r_{1}+r_{2}\right) K(T)\left\|y_{1}-y_{2}\right\|_{\mathcal{X}_{T}} \\
& \leq C_{T}\left\|y_{0,1}-y_{0,2}\right\|_{N_{q, \lambda, \infty}^{-\beta}}+4 r K(T)\left\|y_{1}-y_{2}\right\|_{\mathcal{X}_{T}},
\end{aligned}
$$

and then

$$
\left\|y_{1}-y_{2}\right\|_{\mathcal{X}_{T}} \leq \frac{C_{T}}{1-4 r K(T)}\left\|y_{0,1}-y_{0,2}\right\|_{N_{q, \lambda, \infty}^{-\beta}},
$$

which implies the desired continuity of the data-solution map.

\section{References}

[1] A. C. Eringen, Theory of micropolar fluids. J. Math. Mech. 16 (1966) 1-18.

[2] L.C.F. Ferreira, J.C. Precioso, Existence and asymptotic behaviour for the parabolicparabolic Keller-Segel system with singular data, Nonlinearity 24 (5) (2011), 1433-1449.

[3] L. C. F. Ferreira, E. J. Villamizar-Roa, Micropolar fluid system in a space of distributions and large time behavior, J. Math. Anal. Appl 332 (2007), 1424-1444.

[4] G. Lukaszewicz, Micropolar Fluids, theory and applications, modeling and simulation in science, Engineering and Technology, Birkhäuser, Boston, (1999).

[5] A.L. Mazzucato, Besov-Morrey spaces: function space theory and applications to non-linear PDE, Trans. Amer. Math. Soc. 355 (4) (2003), 1297-1364.

[6] H. Kozono and Y. Hideo, Semilinear heat equations and the Navier-Stokes equation with distributions in new function spaces as initial data, Comm. Partial Differential Equations 19 (5-6) (1994), 959-1014. 